\title{
Redox Properties of Mononuclear Dicarbonyl and Carbonylphosphine Rhodium (I) Complexes Containing $\beta$-diketonate Ligands
}

\author{
Galina V. Burmakina ${ }^{a, b}$, Victor V. Verpekin ${ }^{a}$, \\ Dmitry V. Zimonina, Oleg S. Chudina, \\ Tatyana S. Nedelina ${ }^{b}$ and Anatoly I. Rubaylo ${ }^{a, b}$ \\ anstitute of Chemistry and Chemical Technology SB RAS \\ FRC "Krasnoyarsk Science Center SB RAS" \\ 50/24 Akademgorodok, Krasnoyarsk, 660036, Russia \\ ${ }^{b}$ Siberian Federal University \\ 79 Svobodny, Krasnoyarsk, 660041, Russia
}

Received 11.06.2019, received in revised form 26.07.2019, accepted 30.08.2019

The redox properties of mononuclear dicarbonyl and carbonylphosphine rhodium (I) complexes $(C O)(L) R h\left(R C(O) C H C(O) R^{\prime}\right)$ with chelate $\beta$-diketonate ligands $\left(L=C O, P P h_{3} ; R, R\right.$ ' $=M e, P h ; C F_{3}$, $\left.\mathrm{C}_{4} \mathrm{H}_{3} \mathrm{~S}\right)$ were studied by electrochemical methods at platinum, glassed carbon and dropping mercury electrodes in acetonitrile. Schemes of their redox reactions were established.

Keywords: redox properties, electrochemistry, rhodium, carbonyl, carbonylphosphine, $\beta$-diketonate ligands.

Citation: Burmakina G.V., Verpikin V.V., Zimonin D.V., Chudin O.S., Nedelina T.S., Rubaylo A.I. Redox properties of mononuclear dicarbonyl and carbonylphosphine rhodium (I) complexes containing $\beta$-diketonate ligands, J. Sib. Fed. Univ. Chem., 2019, 12(3), 413-422. DOI: 10.17516/1998-2836-0138.

(c) Siberian Federal University. All rights reserved

* Corresponding author E-mail address: bgvicct@gmail.com,bgv@akadem.ru 


\title{
Окислительно-восстановительные свойства
}

\author{
моноядерных дикарбонильных
}

и карбонилфосфиновых комплексов родия (I), содержащих $\beta$-дикетонатные лиганды

\section{Г.В. Бурмакина ${ }^{\mathrm{a}, \sigma^{*}}$, В.В. Верпекин ${ }^{\mathrm{a}}$, Д.В. Зимонин ${ }^{\mathrm{a}}$, О.С. Чудин ${ }^{\text {a }}$ Т.С. Неделина ${ }^{\text {, }, ~ А . И . ~ Р у б а и ̆ л о, ~}$ ${ }^{a}$ Институт химии и химической технологии СО РАН ФИЦ «Красноярский научный ичентр СО РАН» Россия, 660036, Красноярск, Академгородок,50/24 \\ ${ }^{6}$ Сибирский федеральный университет Россия, 660041, Красноярск, пр. Свободньй, 79}

Электрохимическими методами изучень редокс-свойства моноядерных дикарбонильных и карбонилфосфиновых комплексов родия (I) типа (CO) $(L) R h(R C(O) C H C(O) R$ ') с хелатными $\beta$-дикетонатными лигандами ( $\left.L=C O, P \mathrm{Ph}_{3} ; R, R^{\prime}=\mathrm{Me}, \mathrm{Ph} ; \mathrm{CF}_{3}, C_{4} H_{3} S\right)$ на платиновом, стеклоуглеродном и ртутном капельном электродах в ацетонитриле. Установлены схемы их редокс-реакичий.

Ключевые слова: окислительно-восстановительные свойства, электрохимия, родий, карбонильные, карбонилфосфиновые, $\beta$-дикетонатные лигандыл.

\section{Введение}

Моноядерные дикарбонильные и карбонилфосфиновые комплексы родия (I) с бидентатными $\beta$-дикетонатными лигандами являются эффективными гомогенными катализаторами гидроформилирования олефинов [1-6]. В их основе, как и в других каталитических процессах с участием родия (I) [7-13], лежит способность плоскоквадратных комплексов родия (I) к окислительному присоединению различных органических субстратов с образованием октаэдрических комплексов родия (III), а также к образованию вакантного координационного места за счет элиминирования одного из лигандов. В связи с этим в настоящее время все большее внимание уделяется изучению механизмов окисления с участием комплексов родия (I) и прогнозированию поведения этих соединений в каталитических редокс-реакциях с органическими субстратами в зависимости от их лигандного окружения [1-13].

Электрохимическое окисление моноядерных карбонильных комплексов родия (I) с $\beta$-дикетонатными и фосфорсодержащими лигандами хорошо изучено в ацетонитриле и дихлорметане [14-20]. В то же время их восстановление, а также электрохимическое поведение дикарбонильных комплексов родия (I) с $\beta$-дикетонатными лигандами, кроме ацетилацетонатного, практически не исследованы. 


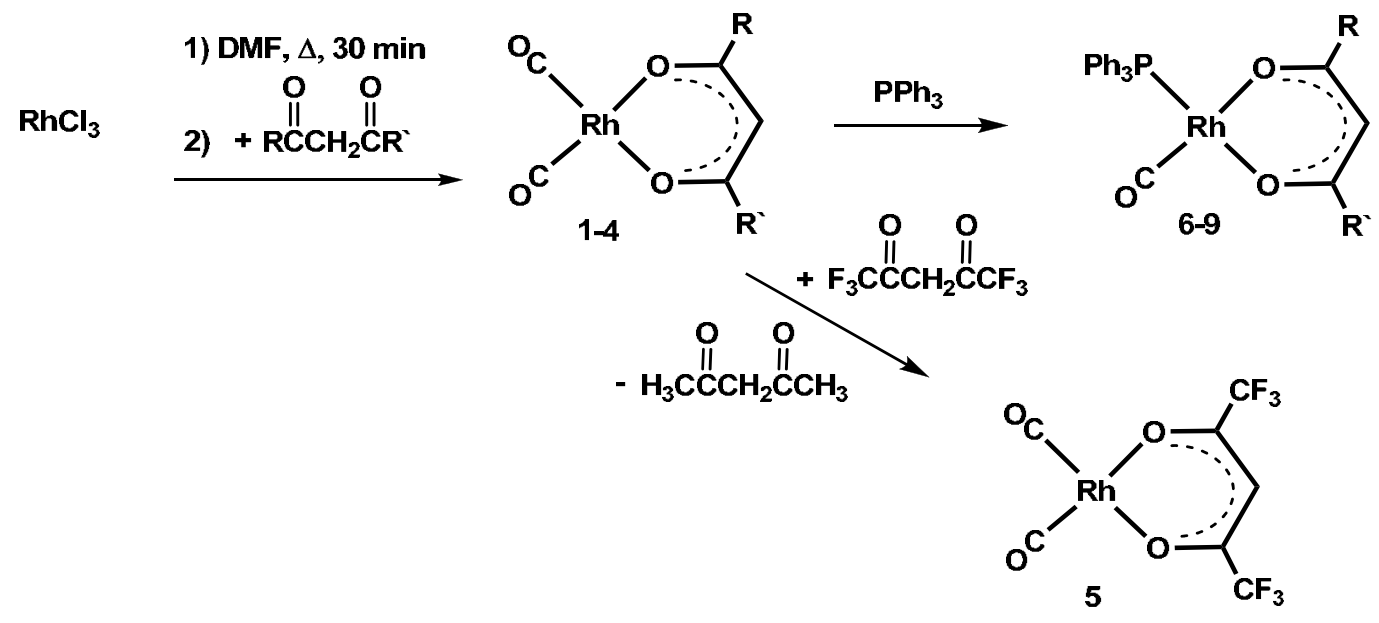

Рис. 1. Схема синтеза комплексов типа (CO)(L)Rh(RC(O)CHC(O)R')

Fig.1. Scheme of the $(\mathrm{CO})(\mathrm{L}) \mathrm{Rh}(\mathrm{RC}(\mathrm{O}) \mathrm{CHC}(\mathrm{O}) \mathrm{R}$ ') complexes synthesis

Настоящая работа посвящена изучению окислительно-восстановительных свойств моноядерных дикарбонильных и для сравнения карбонилфосфиновых комплексов родия (I) типа $(\mathrm{CO})(\mathrm{L}) \mathrm{Rh}(\mathrm{RC}(\mathrm{O}) \mathrm{CHC}(\mathrm{O}) \mathrm{R}$ ') с хелатными $\beta$-дикетонатными лигандами: $\mathrm{L}=\mathrm{CO}$, $\mathrm{R}=\mathrm{R}^{\prime}=\mathrm{Me}(\mathbf{1})$ (acac); R = Me, R' = Ph (2) (ba); R = R' = Ph (3) (dbm), R = $\mathrm{CF}_{3}, \mathrm{R}^{\prime}=\mathrm{C}_{4} \mathrm{H}_{3} \mathrm{~S}(\mathbf{4})$ (tta), $\mathrm{R}=\mathrm{R}^{\prime}=\mathrm{CF}_{3}$ (5) (fta); $\mathrm{L}=\mathrm{PPh}_{3}, \mathrm{R}=\mathrm{R}^{\prime}=\mathrm{Me}$ (6) (acac); $\mathrm{R}=\mathrm{Me}, \mathrm{R}^{\prime}=\mathrm{Ph}$ (7) (ba); R = R' = Ph (8) (dbm), R = $\mathrm{CF}_{3}, \mathrm{R}^{\prime}=\mathrm{C}_{4} \mathrm{H}_{3} \mathrm{~S}$ (9) (tta), методами полярографии и циклической вольтамперометрии в ацетонитриле на ртутном капельном (р.к.э), платиновом (Pt) и стеклоуглеродном (CУ) электродах и установлению влияния природы лигандов на их редокс-свойства.

Комплексы 1-4 синтезированы с применением общего подхода, основанного на реакциях диметиламмония дикарбонилдихлорородоната $\left[\mathrm{Rh}(\mathrm{CO})_{2} \mathrm{Cl}_{2}\right]\left[\mathrm{NH}_{2} \mathrm{Me}_{2}\right]$ [21] с соответствующими $\beta$-дикетонами [22]. Соединение 5 получено по реакции обмена ацетилацетонатного лиганда в $\mathrm{Rh}(\mathrm{acac})(\mathrm{CO})_{2}(\mathbf{1})$ на $1,1,1$-трифторацетилацетон. По реакциям 1-4 с трифенилфосфином были синтезированы соответствующие карбонилфосфиновые комплексы 6-9. Схемы реакций, по которым были получены изученные в работе соединения, приведены на рис. 1.

Для установления схем редокс-реакций комплексов 1-9 в таких же экспериментальных условиях изучены редокс-свойства 1,3-замещенных $\beta$-дикетонов $\mathrm{RC}(\mathrm{O}) \mathrm{CHC}(\mathrm{OH}) \mathrm{R}$, где $\mathrm{R}=\mathrm{R}^{\prime}=\mathrm{Me}(\mathbf{1 0}) ; \mathrm{R}=\mathrm{Me}, \mathrm{R}^{\prime}=\mathrm{Ph}(\mathbf{1 1}) ; \mathrm{R}=\mathrm{R}^{\prime}=\mathrm{Ph}(\mathbf{1 2}) ; \mathrm{R}=\mathrm{CF}_{3}, \mathrm{R}^{\prime}=\mathrm{C}_{4} \mathrm{H}_{3} \mathrm{~S}(\mathbf{1 3}), \mathrm{R}=\mathrm{R}^{\prime}=\mathrm{CF}_{3}(\mathbf{1 4})$ и $\mathrm{PPh}_{3}(\mathbf{1 5})$. Электрохимия $\beta$-дикетонов подробно исследована в работе [23].

\section{Экспериментальная часть}

Комплексы 1-9 синтезированы по методикам [21, 22]. Электрохимические измерения проводили в свежеприготовленных растворах соединений в ацетонитриле, очищенном по известной методике [24], с добавлением $0.1 \mathrm{M} \mathrm{Et}_{4} \mathrm{NBF}_{4}$ в качестве фонового электролита, в атмосфере аргона при комнатной температуре. Полярограммы и циклические вольтамперограммы (ЦВА) регистрировали на потенциостате ІРС-Pro М (ООО НТФ «Вольта», Санкт-Петербург, Россия). 
В качестве рабочих электродов ${ }^{1}$ использовали ртутный капельный электрод с принудительным отрывом капель $(\mathrm{m}=3.6 \mathrm{mг} / \mathrm{c}, \tau=0.23 \mathrm{c})$ и стационарные: платиновый $(\mathrm{d}=1$ мм) или стеклоуглеродный $(\mathrm{d}=5$ мм) в тефлоновых корпусах $(\mathrm{d}=10 \mathrm{~mm})$. Электродом сравнения служил полуэлемент $\mathrm{Ag} / 0.1 \mathrm{M} \mathrm{AgNO}_{3}$ в $\mathrm{MeCN}$, соединенный с ячейкой электролитическим мостом, заполненным фоновым электролитом, через капилляр Луггина. В качестве вспомогательного электрода применяли платиновую спираль, помещенную в стеклянную трубку с пористым фильтром. Число электронов, участвующих в каждой редокс-стадии, определяли сравнением высот волн исследуемых соединений с высотой волны обратимого одноэлектронного окисления ферроцена.

\section{Результаты и обсуждение}

Полученные электрохимические характеристики моноядерных дикарбонильных и карбонилфосфиновых комплексов родия (I) с бидентатными $\beta$-дикетонатными лигандами 1-9, $\beta$-дикетонов 10-14 и $\mathrm{PPh}_{3}(15)$ приведены в таблице, пример типичной циклической вольтамперограммы комплекса 2 и Нba (11) на СУ-электроде - на рис. 2.

Как видно из данных таблицы и рис. 26, для комплексов 1-9 на различных электродах наблюдаются волны окисления и восстановления, значения редокс-потенциалов которых сильно зависят от материала рабочих электродов.

Окисление комплексов 1-9 на Pt и СУ-электродах протекает в одну двухэлектронную стадию. Значения $\mathrm{E}_{1 / 2}$ окисления моноядерных дикарбонильных комплексов родия (I) с

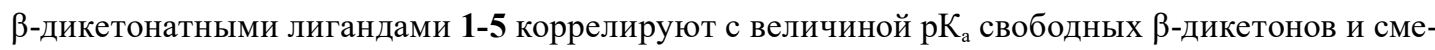
щаются в катодную область в ряду: $\mathbf{5}<\mathbf{4}<\mathbf{1}<\mathbf{2}<\mathbf{3}$ (табл.), в соответствии с увеличением

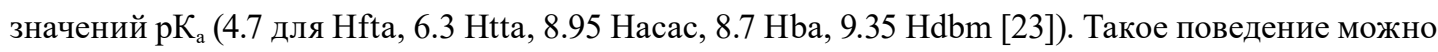
объяснить тем, что введение электроноакцепторных групп, таких как $\mathrm{CF}_{3}$, в $\beta$-дикетонатный лиганд приводит к уменьшению электронной плотности на атоме родия и, соответственно, снижает способность комплексов к их окислению. Наоборот, более легкое окисление комплексов Rh(I) до Rh(III) обусловлено повышением электронной плотности в металлическом центре за счет электронодонорных заместителей в $\beta$-дикетонатном лиганде (с большим значением рК соответствующего $\beta$-дикетона).

Замена одного из карбонильных лигандов в дикарбонильных комплексах родия (I) 1-4 на трифенилфосфиновый лиганд приводит к смещению потенциалов окисления их карбонилфосфиновых аналогов 6-9 в катодную область (табл.) в соответствии с большей электронодонорной способностью $\mathrm{PPh}_{3}$ лиганда по сравнению с СО. При этом общая тенденция влияния заместителей $\mathrm{R}, \mathrm{R}$ ' в $\beta$-дикетонатном лиганде на значения $\mathrm{E}_{1 / 2}$ окисления комплексов 1-9 сохраняется.

Механизм электрохимического окисления моноядерных карбонилфосфиновых комплексов родия (I) с $\beta$-дикетонатными лигандами в различных растворителях подробно изучен в работах $[14,17,19]$. Показано, что необратимое двухэлектронное окисление плоскоквадратных

\footnotetext{
Использование различных рабочих электродов позволяет исследовать процессы окисления и восстановления соединений в более широкой области потенциалов. Так, рабочая область измерения потенциалов в ацетонитриле (относительно $\mathrm{Ag} / 0.1 \mathrm{M} \mathrm{AgNO}_{3}$ в $\mathrm{MeCN}$ ) на р.к.э. находится в пределах от 0.30 до $-3.20 \mathrm{~B}$, на $\mathrm{Pt}$ и СУ электродах от 2.0 до -2.2 В и от 2.0 до -2.6 В соответственно.
} 
Таблица. Электрохимические характеристики моноядерных комплексов родия (I), $\beta$-дикетонов и $\mathrm{PPh}_{3}$ $\left(\mathrm{MeCN}, 0.1 \mathrm{M} \mathrm{Et}_{4} \mathrm{NBF}_{4}, 2\right.$ мM, $\mathrm{Ag} / 0.1 \mathrm{M} \mathrm{AgNO}_{3}$ в $\left.\mathrm{MeCN}\right)$

Table. Electrochemical data of mononuclear rhodium (I) complexes, $\beta$-diketones and $\mathrm{PPh}_{3}\left(\mathrm{MeCN}, 0.1 \mathrm{M} \mathrm{Et}_{4} \mathrm{NBF}_{4}\right.$, $2 \mathrm{mM}, \mathrm{Ag} / 0.1 \mathrm{M} \mathrm{AgNO}_{3}$ in $\left.\mathrm{MeCN}\right)$

\begin{tabular}{|c|c|c|c|c|c|}
\hline \multirow{3}{*}{ Соединение } & \multicolumn{5}{|c|}{$\mathrm{E}_{1 / 2}, \mathrm{~B}(\mathrm{n})$} \\
\hline & \multicolumn{2}{|c|}{$\mathrm{Pt}$} & \multicolumn{2}{|c|}{ CY } & \multirow{2}{*}{$\begin{array}{c}\text { Р.к.э. } \\
\text { Red }\end{array}$} \\
\hline & $\mathrm{Ox}$ & Red & $\mathrm{Ox}$ & Red & \\
\hline $\mathrm{Rh}(\mathrm{CO})_{2}(\mathrm{acac})(\mathbf{1})$ & - & $-1.98(1)$ & $0.66(2)^{\mathrm{a}}$ & $\begin{array}{l}-2.04(1)^{\mathrm{a}} \\
-2.60(<1)\end{array}$ & $\begin{array}{l}-2.04(1) \\
-2.65(1)\end{array}$ \\
\hline $\mathrm{Rh}(\mathrm{CO})\left(\mathrm{PPh}_{3}\right)(\mathrm{acac})(6)$ & $0.64(2)$ & - & $0.39(2)^{\mathrm{a}}$ & $\begin{array}{l}-2.25(1)^{\mathrm{a}} \\
-2.62(<1)\end{array}$ & $\begin{array}{l}-2.28(1) \\
-2.71(1) \\
-3.00(1)\end{array}$ \\
\hline $\mathrm{Rh}(\mathrm{CO})_{2}(\mathrm{ba})(\mathbf{2})$ & - & - & $0.64(2)^{\mathrm{a}}$ & $\begin{array}{l}-2.00(1)^{\mathrm{a}} \\
-2.27(<1)\end{array}$ & $\begin{array}{c}-2.00(1) \\
-2.30(<1)\end{array}$ \\
\hline $\mathrm{Rh}(\mathrm{CO})\left(\mathrm{PPh}_{3}\right)(\mathrm{ba})(7)$ & $0.62(2)$ & - & $0.36(2)^{\mathrm{a}}$ & $-2.20(1)^{\mathrm{a}}$ & $\begin{array}{l}-2.20(1) \\
-2.90(1)\end{array}$ \\
\hline $\mathrm{Rh}(\mathrm{CO})_{2}(\mathrm{dbm})(\mathbf{3})$ & - & $-1.81(1)$ & $0.60(2)^{\mathrm{a}}$ & $-1.86(1)^{\mathrm{a}}$ & $-1.95(1)$ \\
\hline $\mathrm{Rh}(\mathrm{CO})\left(\mathrm{PPh}_{3}\right)(\mathrm{dbm})(\mathbf{8})$ & $0.58(2)$ & - & $0.33(2)^{\mathrm{a}}$ & $-2.17(1)^{\mathrm{a}}$ & $\begin{array}{l}-2.27(1) \\
-2.94(1)\end{array}$ \\
\hline $\mathrm{Rh}(\mathrm{CO})_{2}(\mathrm{tta})(\mathbf{4})$ & - & $-1.54(1)$ & $0.73(2)^{\mathrm{a}}$ & $-1.61(1)^{\mathrm{a}}$ & $-1.79(1)$ \\
\hline $\mathrm{Rh}(\mathrm{CO})\left(\mathrm{PPh}_{3}\right)(\mathrm{tta})(\mathbf{9})$ & $0.71(2)$ & $-1.80(1)$ & $0.52(2)^{\mathrm{a}}$ & $-1.87(1)^{\mathrm{a}}$ & $\begin{array}{l}-1.89(1) \\
-2.94(1)\end{array}$ \\
\hline $\mathrm{Rh}(\mathrm{CO})_{2}(\mathrm{fta})(\mathbf{5})$ & $1.00(2)$ & $-0.93(1)$ & $0.96(2)^{\mathrm{a}}$ & $-1.02(1)^{\mathrm{a}}$ & $\begin{array}{c}-1.32(1) \\
-1.81(<1)\end{array}$ \\
\hline Hacac (10) & - & - & - & - & $-2.71(1)$ \\
\hline Hba (11) & - & $-2.00(1)$ & - & $-2.17(1)$ & $-2.20(1)$ \\
\hline $\operatorname{Hdbm}(\mathbf{1 2})$ & - & $-1.90(1)$ & - & $-1.98(1)$ & $-2.02(1)$ \\
\hline Htta (13) & - & $-1.59(1)^{\mathrm{a}}$ & - & $-1.59(1)^{\mathrm{a}}$ & $-1.70(1)$ \\
\hline Hfta (14) & - & - & - & $-1.28(1)$ & $-1.40(1)$ \\
\hline $\mathrm{PPh}_{3}(15)$ & - & - & - & - & $-2.95(1)$ \\
\hline
\end{tabular}

Примечание. $\mathrm{n}$ - число электронов, участвующих в электрохимической стадии (знак «<» означает, что высота волны исследуемого соединения меньше, чем высота одноэлектронной волны); а - квазиобратимая стадия.

Notes: $\mathrm{n}$ - the number of electrons transferred in a particular electrochemical stage (the sign " $<$ " indicates that the wave height is smaller than the height of the one-electron wave); a - quasi-reversible stage.

комплексов родия (I) приводит к образованию октаэдрических комплексов родия (III). При этом происходит присоединение двух молекул растворителя к образующемуся комплексу родия (III) по схеме (рис. 3) [19].

Восстановление дикарбонильных и карбонилфосфиновых комплексов родия (I) 1-9 в работе изучено на трех различных электродах (табл., рис. 2, 4). Установлено, что в отличие от электрохимического окисления восстановление комплексов 1-9 на всех трех электродах протекает с присоединением на первой стадии только одного электрона (табл., рис. 2). Значения $\mathrm{E}_{1 / 2}$ первых стадий восстановления дикарбонильных комплексов 1-5 смещаются в катодную область в следующем порядке: $\mathbf{1}>\mathbf{2}>\mathbf{3}>\mathbf{4}>\mathbf{5}$ (табл.), при этом замена одного из $\pi$-электроноакцепторных карбонильных лигандов в комплексах 1-4 на б-электронодонорный трифенилфосфиновый ли- 


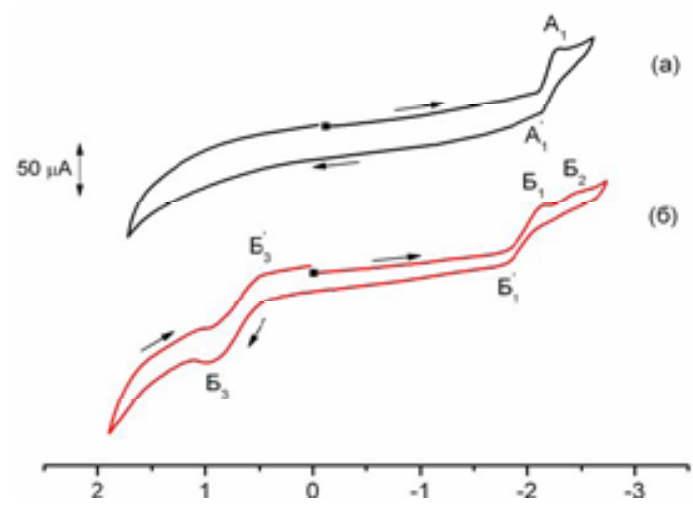

Рис. 2. Циклические вольтамперограммы: (a) $\mathrm{Hba}(\mathbf{1 1})$, (б) $\mathbf{R h}(\mathrm{CO})_{2}$ (ba) (2) (CУ, MeCN, $0.1 \mathrm{M} \mathrm{Et}_{4} \mathrm{NBF}_{4}$, $\mathrm{Ag} / 0.1 \mathrm{M} \mathrm{AgNO}_{3}$ в $\left.\mathrm{MeCN}, \mathrm{C}=2 \mathrm{MM}, v=25 \mathrm{mB} / \mathrm{c}\right)$

Fig. 2. Cyclic voltammograms of: (a) $\mathrm{Hba}(\mathbf{1 1})$, (б) $\mathbf{R h}(\mathrm{CO})_{2}$ (ba) (2) (GC, $\mathrm{MeCN}, 0.1 \mathrm{M} \mathrm{Et}_{4} \mathrm{NBF}_{4}, \mathrm{Ag} / 0.1 \mathrm{M} \mathrm{AgNO}_{3}$ in $\mathrm{MeCN}, \mathrm{C}=2 \mathrm{mM}, v=25 \mathrm{mV} / \mathrm{s}$ )<smiles>[R]C1=CC([R])O[R1](C)([PbH])O1</smiles>
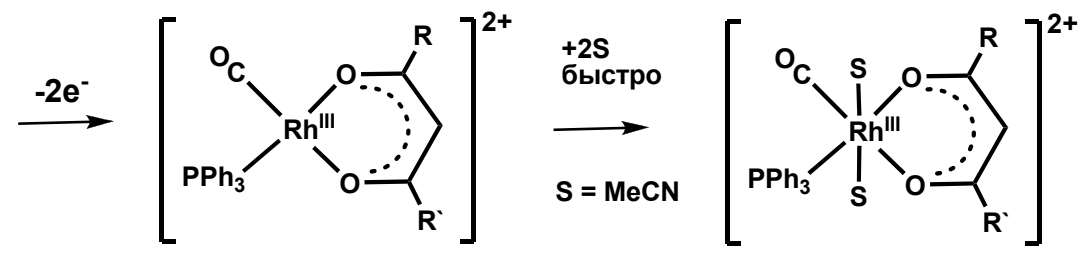

Рис. 3. Схема окисления комплексов родия (I)

Fig. 3. Oxidation scheme of rhodium (I) complexes

ганд приводит не к изменению этой зависимости, а только к смещению потенциалов восстановления комплексов 6-9 в катодную область по сравнению с 1-4 (табл.). На циклических вольтамперограммах и полярограммах комплексов 1, 2 и 6, зафиксированных на СУ и р.к.э. электродах, наблюдается вторая волна восстановления (табл., рис. 26 , пик $\mathrm{Б}_{2}$, рис. 4, пик $\mathrm{A}_{2}$ ), значение $\mathrm{E}_{1 / 2}$ которой близко к потенциалу восстановления соответствующего $\beta$-дикетона (табл., рис. $2 a$, пик $\mathrm{A}_{1}$ ), что свидетельствует об образовании аниона $\beta$-дикетона в результате одноэлектронного восстановления комплексов 1 и 6.

Для подтверждения этого предположения изучены редокс-реакции комплексов 1, 2 и 6 в присутствии фенола, используемого в качестве донора протонов (рис. 4). Добавление фенола к раствору комплексов 1, 2 и 6 (в соотношении 1:1) не приводит к изменению их циклических вольтамперограмм в процессе окисления (в области изменения потенциалов от 0.0 В до 2.0 В). На циклических вольтамперограммах комплексов 1, 2 и 6 при их восстановлении (в области изменения потенциалов от 0.0 В до -2.8 В) в присутствии фенола наблюдается увеличение высоты второй волны восстановления комплексов и смещение значения ее $\mathrm{E}_{1 / 2}$ в анодную область (рис. 4, пик $\mathrm{Б}_{2}$ ) по сравнению с ЦВА их восстановления без фенола (рис. 4, пик $\mathrm{A}_{2}$ ), что свидетельствует об участии протона на второй стадии восстановления 1,2 и 6 и образовании соответствующего свободного $\beta$-дикетона, который восстанавливается при более положительных значениях потенциалов по сравнению с анионом. Кроме того, после одноэлектронного восста- 


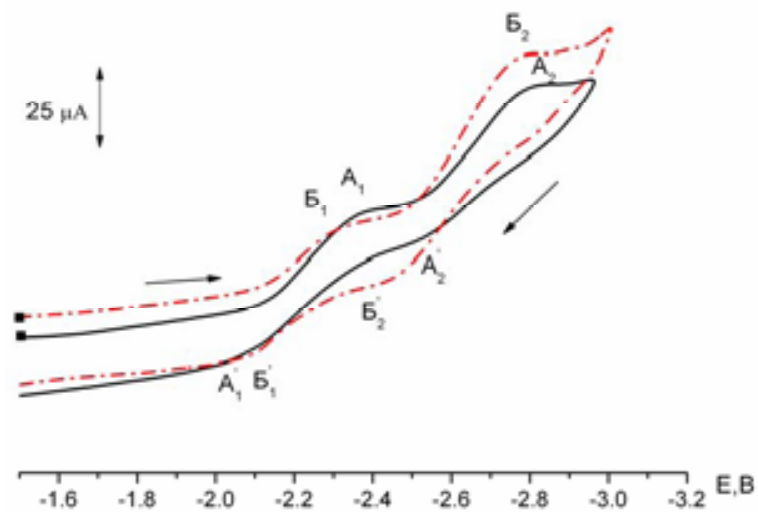

Рис. 4. Циклические вольтамперограммы: сплошная линия - $\mathbf{R h}(\mathrm{CO})\left(\mathrm{PPh}_{3}\right)(\mathrm{acac})(\mathbf{6})$, штрихпунктирная линия - $\mathbf{R h}(\mathrm{CO})\left(\mathrm{PPh}_{3}\right)(\mathrm{acac})(\mathbf{6})$ с добавлением фенола в соотношении 1:1 (CУ, MeCN, $0.1 \mathrm{M} \mathrm{Et}_{4} \mathrm{NBF}_{4}$, $\mathrm{Ag} / 0.1 \mathrm{M} \mathrm{AgNO}$ в $\mathrm{MeCN}, \mathrm{C}=2 \mathrm{MM}, v=25 \mathrm{MB} / \mathrm{c})$

Fig. 4. Cyclic voltammograms of: solid line $-\mathbf{R h}(\mathrm{CO})\left(\mathrm{PPh}_{3}\right)(\mathrm{acac})(\mathbf{6})$, dash bot line $\mathbf{R h}(\mathrm{CO})\left(\mathrm{PPh}_{3}\right)(\mathrm{acac})(\mathbf{6})$ with addition of phenol in a ratio of 1:1 $\left(\mathrm{GC}, \mathrm{MeCN}, 0.1 \mathrm{M} \mathrm{Et}_{4} \mathrm{NBF}_{4}, \mathrm{Ag} / 0.1 \mathrm{M} \mathrm{AgNO}_{3}\right.$ in $\mathrm{MeCN}, \mathrm{C}=2 \mathrm{mM}, v=$ $25 \mathrm{mV} / \mathrm{s}$ )

новления комплексов 1-9 на поверхности ртутного и Рt-электродов появляется темно-серый налет, что свидетельствует об образовании родия (0) в результате восстановления комплексов родия (I).

Следует отметить, что первые волны окисления и восстановления на СУ-электроде комплексов 1-9 квазиобратимы. Как видно на рис. 2, отношение предельных токов катодного и анодного пиков стадии окисления комплекса $2 \mathrm{I}_{\mathrm{pc}} \mathrm{I}_{\mathrm{pa}}=0.59$ (рис. 26 , пики $\mathrm{b}_{3}^{\prime}, \mathrm{b}_{3}$ ), анодного и катодного пиков первой стадии восстановления $\mathrm{I}_{\mathrm{pa}} / \mathrm{I}_{\mathrm{pc}}=0.44$ (рис. 26 , пики $\mathrm{b}_{1}^{\prime}, \mathrm{5}_{1}$ ) соответствуют квазиобратимому процессу переноса электрона [25].

Таким образом, электрохимическое изучение моноядерных дикарбонильных и карбонилфосфиновых комплексов родия (I) с $\beta$-дикетонатными лигандами 1-9 показало, что их редокспревращения квазиобратимы и протекают по следующей схеме (рис. 5).

Одноэлектронное квазиобратимое восстановление как дикарбонильных 1-5, так и карбонилфосфиновых 6-9 комплексов приводит к образованию аниона, который в дальнейшем распадается до родия (0) и аниона соответствующего $\beta$-дикетона (рис. 6).

Двухэлектронное квазиобратимое окисление дикарбонильных комплексов 1-5 протекает по схеме, подобной ранее установленной для карбонилфосфиновых комплексов 6-9 (см. рис. 3),<smiles>[R]C1=CC(C)O[P](C)(C)O1</smiles>

Рис. 5. Схема редокс-превращений комплексов родия (I)

Fig. 5. Redox transformation scheme of rhodium (I) complexes 


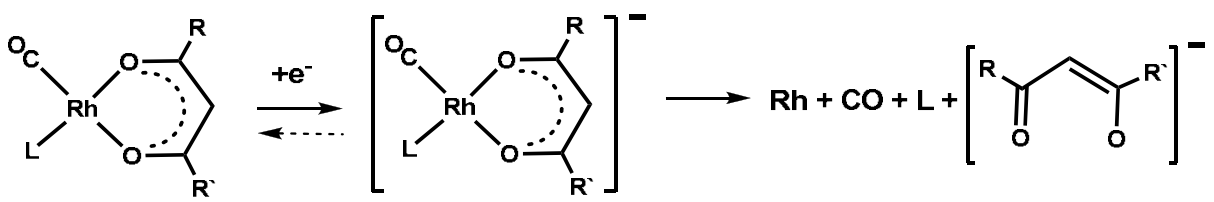

Рис. 6. Схема восстановления комплексов родия (I)

Fig. 6. Reduction scheme of rhodium (I) complexes

однако при более положительных значениях потенциалов по сравнению с 6-9, то есть труднее.

\section{Заключение}

В работе впервые изучены окислительно-восстановительные свойства моноядерных дикарбонильных комплексов родия (I) с хелатными $\beta$-дикетонатными лигандами 1-5 и проведено их сравнение с карбонилфосфиновыми аналогами 6-9. Полученные данные свидетельствуют о том, что использование карбонилфосфиновых комплексов родия (I) в каталических редокс-процессах превращения органических соединений [1-6] предпочтительнее дикарбонильных, поскольку комплексы 6-9 легче окисляются и труднее восстанавливаются по сравнению с 1-5. При этом показано, что значения потенциалов окисления всех изученных комплексов коррелируют с величиной $\mathrm{pK}_{\mathrm{a}}$ соответствующих свободных $\beta$-дикетонов (с увеличением $\mathrm{pK}_{\mathrm{a}} \beta$-дикетона комплексы окисляются легче). Установлены схемы восстановления комплексов 1-9.

\section{Список литературы}

1. Mieczynska E., Trzeciak A.M., Grzybek R., Ziolkowski J.J. New rhodium systems for biphasic hydrogenation and hydroformylation of 1-hexene. J. Mol. Catal. A. 1998. Vol. 123. P. 203212.

2. Pruchnik F.P., Smolenski P., Wajda-Hermanowicz K. Rhodium(I) acetylacetonato complexes with functionalized phosphines. J. Organomet. Chem. 1998. Vol. 570. P. 63-69.

3. Trzeciak A.M., Ziolkowski J.J. Perspectives of rhodium organometallic catalysis. Fundamental and applied aspects of hydroformylation. Coord. Chem. Rev. 1999. Vol. 190-192. P. 883-900.

4. Zhang J., Sun X.-Z., Poliakoff M., George M. W. Study of the reaction of Rh(acac)(CO $)_{2}$ with alkenes in polyethylene films under high-pressure hydrogen and the Rh-catalysed hydrogenation of alkenes. J. Organomet. Chem. 2003. Vol. 678. P. 128-133.

5. Conradie M.M., Conradie J. Stereochemistry of the reaction products of the oxidative addition reaction of methyl iodide to $\left[\mathrm{Rh}\left(\left(\mathrm{C}_{4} \mathrm{H}_{3} \mathrm{~S}\right) \mathrm{COCHCOR}\right)(\mathrm{CO})\left(\mathrm{PPh}_{3}\right)\right]$ : A NMR and computional study. $\mathrm{R}=$ $\mathrm{CF}_{3}, \mathrm{C}_{6} \mathrm{H}_{5}, \mathrm{C}_{4} \mathrm{H}_{3}$ S. Inorg. Chim. Acta. 2009. Vol. 362. P. 519-530.

6. Lazzaronia R., Settambolob R., Alagonac G., Ghio C. Investigation of alkyl metal intermediate formation in the rhodium-catalyzed hydroformylation: experimental and theoretical approaches. Coord. Chem. Rew. 2010. Vol. 254. P. 696-706.

7. Мастерс К. Гомогенный катализ переходными металлами. М.: Мир, 1983. 304 с. [Masters C. Homogeneous Transition-metal Catalysis: A Gentle Art. Moskow.: Mir, 1983, 304 p. (In Russ)] 
8. Эльшенбройх К. Металлоорганическая химия. М.: БИНОМ, 2013. 746 с. [Elshenbroich K. Organometallic chemistry. Moskow: BINOM, 2013. 746 p. (In Russ)]

9. Maitlis P.M., Haynes A., Sunley G.J., Howard M.J. Methanol carbonylation revisited: thirty years on. J. Chem. Soc. Dalton Trans. 1999. P. 2187-2196.

10. Ivanova E. A, Gisdakis P., Nasluzov V.A., Rubailo A.I., Rösch N. Methanol carbonilation catalyzed by the complex $\left[\mathrm{Rh}(\mathrm{CO})_{2} \mathrm{I}_{2}\right]$. Organometallics. 2001. Vol. 20. P. 1161-1174.

11. Kondo M., Kochi T., Kakiuchi F. Rhodium-catalyzed anti-Markovnikov intermolecular hydroalkoxylation of terminal acetylenes. J. Am. Chem. Soc. 2011. Vol. 133(1). P. 32-34.

12. Dang Y., Qu S., Wang Z., Wang X. Mechanism and origins of Z selectivity of the satalytic hydroalkoxylation of alkynes via Rhodium vinylidene complexes to produce enol ethers. Organometallics. 2013. Vol. 32. P. 2804-2813.

13. Warsink S., Kotze P.D.R., Janse van Rensburg J.M., Venter J.A., Otto S., Botha E., Roodt A. Kinetic-mechanistic and solid state study of the iodomethane oxidative addition to and migratory insertion in [Rhodium $(S, O-B d i P T$ or $\left.N, O o x)(\mathrm{CO})\left(\mathrm{PR}^{1} \mathrm{R}^{2} \mathrm{R}^{3}\right)\right]$ complexes. Eur. J. Inorg. Chem. 2018. Vol. 32. P. 3615-3625.

14. Lamprecht D., Lamprecht G.J. Electrochemical oxidation of $\mathrm{Rh}(\mathrm{I})$ to $\mathrm{Rh}(\mathrm{III})$ in rhodium(I) $\beta$-diketonato carbonyl phosphine complexes. Inorg. Chim. Acta. 2000. Vol. 309. P. 72-76.

15. Guedes da Silva M.F.C., Trzeciak A.M., Ziólkowski J.J., Pombeiro A.J.L. Redox potential, ligand and structural effects in rhodium(I) complexes. J. Organomet. Chem. 2001. Vol. 620. P. 174-181.

16. Conradie J., Cameron T.S., Aquino M.A.S., Lamprecht G.J., Swarts J.C. Synthetic, electrochemical and structural aspects of a series of ferrocene-containing dicarbonylbetadiketonato rhodium(I) complexes. Inorg. Chim. Acta. 2005. Vol. 358. P. 2530-2542.

17. Conradie J., Swarts J.C. The relationship between the electrochemical and chemical oxidation of ferrocene-containing carbonyl phosphine B-diketonatorhodium(I) complexes; cytotoxicity of $\left[\mathrm{Rh}(\mathrm{FcCOCHCOPh})(\mathrm{CO})\left(\mathrm{PPh}_{3}\right)\right]$. Eur. J. Inorg. Chem. 2011. Vol. 13. P. 2439-2449.

18. Erasmus J.J.C., Conradie J. Chemical and electrochemical oxidation of [Rh( $\beta$-diketonato) (CO) $\left.\left(\mathrm{P}\left(\mathrm{OCH}_{2}\right)_{3} \mathrm{CCH}_{3}\right)\right]$ : an experimental and DFT study. J. Chem. Soc. Dalton Trans. 2013. Vol. 42. P. $8655-8666$.

19. Ferreira H., Conradie M.M., Conradie J. Electrochemical study of carbonyl phosphine $\beta$-diketonato rhodium(I) complexes. Electrochim. Acta 2013. Vol. 113. P. 519-526.

20. Erasmus E. Synthesis and electrochemistry of p-substituted phenyl diphenylphosphinite rhodium(I) complexes. Polyhedron 2016. Vol. 106. P. 18-26.

21. Serp P., Hernandez M., Kalck P., Richard B. Rhodium (I) and iridium (I) carbonyl complexes. Inorganic synthesis, Vol.34 / J.R. Shapley.: John Wiley \& Sons, Ltd, 2004. P. 121-126.

22. Варшавский Ю.С., Черкасова Т.Г. Простой метод получения ацетилацетонатадикарбонил родия (I). Журн. неорганич. химии. 1967. Т. 12. С. 1709 [Varshavsky Yu.S., Cherkasova T.G. A simple method for preparing acetylacetonatedicarbonyl rhodium (I). Russ. J. Inorg. Chem. 1967. Vol. 12. P. 1709. (In Russ.)].

23. Kuhn, K.G. von Eschwege, Conradie J. Electrochemical and DFT-modeledreduction of enolized 1,3-diketones. Electrochim. Acta. 2011. Vol. 56. P. 6211-6218.

24. Вайсбергер Ф., Проскауэр Э., Диддик Дж., Тупс Э. Органические растворители. Физические свойства и методы очистки. М.: Иностр. лит., 1958. 519 с. [Weisberger F., Proskauer Е., 
Diddik J., Toops E. Organic solvents. Physical properties and purification methods. Mosow: Inostr. lit., 1958. 519 p. (In Russ.)].

25. Gritzner G., Kuta J. Recommendations on reporting electrode potentials in nonaqueous solvents. Pure Applied Chemistry 1984. Vol. 56. P. 461. 